\title{
Higher-derivative harmonic oscillators: stability of classical dynamics and adiabatic invariants
}

\author{
Nicolas Boulanger ${ }^{1, \mathrm{a}}$, Fabien Buisseret ${ }^{2,3, \mathrm{~b}}$, Frédéric Dierick ${ }^{2,4, \mathrm{c}}$, Olivier White ${ }^{5, \mathrm{~d}}$ \\ ${ }^{1}$ Service de Physique de l'Univers, Champs et Gravitation, Research Institute for Complex Systems, Université de Mons-UMONS, Place du Parc \\ 20, 7000 Mons, Belgium \\ ${ }^{2}$ Forme and Fonctionnement Humain Lab, Department of Physical Therapy, CERISIC, Haute Ecole Louvain en Hainaut, 136 rue Trieu Kaisin, \\ 6061 Montignies sur Sambre, Belgium \\ ${ }^{3}$ Service de Physique Nucléaire et Subnucléaire, UMONS Research Institute for Complex Systems, Université de Mons, 20 Place du Parc, 7000 \\ Mons, Belgium \\ ${ }^{4}$ Faculté des Sciences de la Motricité, Université catholique de Louvain, 1 Place Pierre de Coubertin, 1348 Louvain-la-Neuve, Belgium \\ ${ }^{5}$ Université de Bourgogne INSERM-U1093 Cognition, Action, and Sensorimotor Plasticity, Campus Universitaire, BP 27877, 21078 Dijon, France
}

Received: 12 November 2018 / Accepted: 6 January 2019 / Published online: 28 January 2019

(C) The Author(s) 2019

\begin{abstract}
The status of classical stability in higher-derivative systems is still subject to discussions. In this note, we argue that, contrary to general belief, many higher-derivative systems are classically stable. The main tool to see this property are Nekhoroshev's estimates relying on the action-angle formulation of classical mechanics. The latter formulation can be reached provided the Hamiltonian is separable, which is the case for higher-derivative harmonic oscillators. The Pais-Uhlenbeck oscillators appear to be the only type of higher-derivative harmonic oscillator with stable classical dynamics. A wide class of interaction potentials can even be added that preserve classical stability. Adiabatic invariants are built in the case of a Pais-Uhlenbeck oscillator slowly changing in time; it is shown indeed that the dynamical stability is not jeopardised by the time-dependent perturbation.
\end{abstract}

\section{Introduction}

Variational principles based on action functionals of the form $S[x]=\int L(x, \dot{x}) d t$ have a special status in the sense that Newtonian mechanics can be recovered from Lagrangians $L(x, \dot{x})$ depending only on position and velocity. Still, Lagrangians depending on higher time derivatives of the position, i.e, higher-derivative (HD) Lagrangians, are also worth of interest. Let us mention three areas in which HD models are encountered:

\footnotetext{
a e-mail: nicolas.boulanger@umons.ac.be

be-mail: buisseretf@helha.be

ce-mail: dierickf@helha.be

d e-mail: olivier.white@u-bourgogne.fr
}

1. Explicit construction of classically stable and unstable HD dynamical systems: A class of classical HD harmonic oscillators was proposed in Sect. II of [1] and is still actively studied nowadays under the name of PaisUhlenbeck (P-U) oscillator, see e.g. Refs. [2-13] and references therein for recent contributions to the field;

2. Renormalizability of HD field theories: In their pioneering work [1], Pais and Uhlenbeck addressed the issue of renormalizability in field theory through the inclusion of HD terms. HD gravities, like Weyl gravity, are promising renormalisable models of quantum gravity, see the seminal paper $[14,15]$ and recent references in $[16,17]$. These HD models bring in the Einstein-Hilbert term upon radiative corrections, see e.g. [18]. They are also interesting in the context of cosmology and supergravity, see [19-22] and Refs. therein;

3. HD effective dynamics of voluntary human motions: The underlying dynamics of such motions is expected to involve HD variational principles such as minimal jerk, see e.g. Refs. [23-27]. In this case, higher derivative terms may be thought of as a way to account for an intrinsic nonlocality (in time) of planified motion: Human motor control may indeed add memory effects to standard Newtonian dynamics, which may be translated into a HD effective action.

A key feature of classical HD dynamics is that the energy has no definite sign, as it is readily observed from the general structure of HD Hamiltonians [28]. The presence of HD terms may lead to unbounded trajectories at the classical level - an explicit case is built in [2] - and to loss of unitarity at the quantum level [10]. However several cases are known for 
which classical trajectories are bounded and unitarity is preserved at the quantum level [10]. Having these recent results in mind, we think that providing a general method in order to assess classical stability of HD models is worth of interest. This is the main goal of the present paper, in which we focus on the case of HD harmonic oscillators. In the case of perturbed harmonic oscillators, an important body of works concerning their stability has been produced $[29,30]$ that seems to have gone unnoticed by the HD community, so far. In a very specific case of a $\mathrm{P}-\mathrm{U}$ oscillator with at most two time derivatives in the Lagrangian, Pagani et al. proved stability under a general class of cubic and quartic interactions [31].

The present paper is organised as follows. The Lagrangian (Sect. 2) and Hamiltonian (Sect. 3) formulations of HD harmonic oscillators are reviewed and the necessary conditions for their classical trajectories to be bounded are established. The dynamics is then formulated in terms of the action-angle coordinates in Sect. 4 and adiabatic invariants are computed. Finally, classical stability against time-dependent perturbations is discussed by using Nekhoroshev's estimates [32,33].

\section{Lagrangian formulation}

In this section we review the Lagrangian formulation of HD classical systems with finitely many degrees of freedom, in essentially the way that was presented long ago by Ostrogradki [28]. We then review the Pais-Uhlenbeck parametrisation [1] of HD Lagrangians.

\subsection{Generalities}

Let $L\left(x^{(0)}, x^{(1)}, \ldots, x^{(N)}\right)$ be a Lagrangian depending on the $N$ first derivatives of the dynamical variables $x(t):=x^{(0)}(t)$, $x^{(1)}(t):=\dot{x}(t)$ etc. possibly upon adding total derivatives in order to lower as much as possible the order of derivatives of $x(t)$. The action, evaluated between time $t_{1}$ and time $t_{2}$, is $S[x]=\int_{t_{1}}^{t_{2}} L d t$. Hamilton's variational principle $\delta S=0$ implies the equations of motion

$0=\frac{\delta L}{\delta x} \equiv \sum_{j=0}^{N}\left(-\frac{d}{d t}\right)^{j} \frac{\partial L}{\partial x^{(j)}}$,

together with the vanishing of the boundary terms

$$
\begin{aligned}
& \left.\sum_{i=0}^{N-1} \delta x^{(i)} p_{i}\right|_{t_{1}} ^{t_{2}}=0, \\
& p_{i}:=\frac{\delta L}{\delta x^{(i+1)}} \equiv \sum_{j=0}^{N-i-1}\left(-\frac{d}{d t}\right)^{j} \frac{\partial L}{\partial x^{(i+j+1)}}, \\
& i \in\{0, \ldots, N-1\} .
\end{aligned}
$$

One chooses to cancel the above boundary terms by imposing the following conditions at the boundaries of the integration domain:

$\delta x^{(i)}\left(t_{2}\right)=0=\delta x^{(i)}\left(t_{1}\right), \quad \forall i \in\{0, \ldots, N-1\}$.

It amounts to declaring that the initial data needed for solving the equation (1) is given by the values of $x^{(0)}, x^{(1)}, \ldots$, $x^{(N-2)}$ and $x^{(2 N-1)}$ at initial time $t_{1}$. Indeed, provided one assumes the regularity condition

$\frac{\partial^{2} L}{\partial x^{(N)} \partial x^{(N)}} \neq 0$

the above initial data give a well-posed Cauchy problem for the ordinary differential equation (1).

\subsection{Toy model}

The simple HD harmonic oscillator

$L_{\text {toy }}=\frac{\lambda}{2}\left(x^{(N)}\right)^{2}-\frac{(-)^{N+1}}{2} \lambda \beta^{2 N} x^{2}$,

with $N \in \mathbb{N}_{0}$ and $\lambda, \beta \in \mathbb{R}^{+}$can be used to illustrate some features of HD dynamics. It reduces to the standard harmonic oscillator for $N=1$, in which case $\lambda$ is a mass-parameter. The case $N=2$ has already been used as a toy model in [34].

The equation of motion (1) reads

$x^{(2 N)}+\beta^{2 N} x=0$,

and its classical solution is given by

$$
\begin{aligned}
x(t) & =\sum_{j=0}^{2 N-1} A_{j} \mathrm{e}^{\beta_{j} t} \text { with } \beta_{j}=\beta \mathrm{e}^{i \theta_{j}} \text { and } \\
\theta_{j} & =\frac{\pi}{2 N}+\frac{j \pi}{N} .
\end{aligned}
$$

It can be observed from $x(t)$ that the allowed trajectories go beyond a standard periodic motion since, up to an appropriate choice of $A_{j}$, there may appear:

- unbounded when $\operatorname{Re} \beta_{j}>0$. This occurs for $j$ such that $0<2 j+1<N$ and $3 N<2 j+1 \leqslant 4 N-1$;

- damped when $\operatorname{Re} \beta_{j}<0$. This occurs for $j$ such that $N<2 j+1<3 N$;

- periodic with period $2 \pi / \beta$ if $\operatorname{Re} \beta_{j}=0$ : This can happen for $N$ odd and the two values $j=(N-1) / 2$ and $j=$ $(3 N-1) / 2$.

The standard case $N=1$ is the only value for which all the possible trajectories are periodic. When $N>1$, damping or "blowing-up" phenomena occur at time scales of order $\beta \cos (\pi / 2 N)$. 


\subsection{General case: Pais-Uhlenbeck oscillator}

Lagrangian (3) always allows for unbounded classical motions. It can be showed that adding intermediate derivatives $x^{(0<i<N)}$ may reduce and even suppress such instabilities. ${ }^{1}$ Lagrangian (3) may be generalised as follows

$L\left(x^{(0)}, x^{(1)}, \ldots, x^{(N)}\right)=\frac{1}{2} \sum_{j=0}^{N} a_{j}\left(x^{(j)}\right)^{2}, \quad a_{j} \in \mathbb{R}$,

and the corresponding equation of motion reads

$\sum_{j=0}^{N}(-)^{j} a_{j} x^{(2 j)}=0$.

The characteristic polynomial of the above differential equation is

$p(\omega)=\sum_{j=0}^{N}(-)^{j} a_{j} \omega^{2 j}$.

If the signs of $a_{i}$ are alternating, i.e. $a_{j}=(-)^{j+1} b_{j}$ with $b_{j} \in \mathbb{R}_{0}^{+}$, then $p(\omega)$ can be factorised under the form $p(\omega) \sim$ $\Pi_{j=1}^{N}\left(\omega^{2}-\left(i \omega_{j}\right)^{2}\right)$ with $\omega_{j} \in \mathbb{R}_{0}$ and all the trajectories $x(t)$ will be bounded. The further choice $a_{0}<0$ is such that a standard potential energy is recovered for $N=1$.

Therefore, replacing $\left(x^{(j)}\right)^{2}$ by $(-)^{j} x x^{(2 j)}$ up to total derivatives, Lagrangian (6) can be rewritten as

$L\left(x^{(0)}, x^{(1)}, \ldots, x^{(N)}\right)=-\frac{1}{2} x \sum_{j=0}^{N} b_{j} x^{(2 j)}$.

Lagrangian (9) is nothing but the $\mathrm{P}-\mathrm{U}$ oscillator [1], originally written under the equivalent form

$L=-\frac{1}{2} x F_{N}\left(\frac{d}{d t}\right) x$ where $F_{N}(D)=\prod_{i=0}^{N-1}\left(1+\frac{D^{2}}{\omega_{i}^{2}}\right)$

where the frequencies $\omega_{i}$ are assumed to be real and distinct. It has been shown in [1] that equal or imaginary frequencies lead to unbounded trajectories so these cases will not be considered in the following.

The solution of the equation of motion related to (10), $F_{N}\left(\frac{d}{d t}\right) x=0$, reads $x(t)=\sum_{k=1}^{N} A_{k} \sin \left(\omega_{k} t+\varphi_{k}\right)$ with $A_{k}, \varphi_{k} \in \mathbb{R}$. All classical trajectories are therefore bounded. Note that $x(t)$ may describe the motion of a given mass in an $N$-body coupled harmonic oscillator whose normal modes have frequencies $\omega_{i}$ : The formal analogy between the $\mathrm{P}-$ $\mathrm{U}$ oscillator and the dynamics of an $N$-body spring-mass system has been explored in the $N=2$ case in Ref. [36].

\footnotetext{
1 Adding degrees of freedom coupled to $x$ is another way of addressing the problem, see. e.g. [35].
}

An equivalent writing of (10) makes use of the oscillator variables

$$
Q_{i}:=\prod_{0=j \neq i}^{N-1}\left(1+\frac{1}{\omega_{j}^{2}} \frac{d^{2}}{d t^{2}}\right) x
$$

and shows that (10) can formally be written as a Lagrangian describing $N$ decoupled harmonic oscillators:

$L=-\frac{1}{2} \sum_{i=0}^{N-1} \eta_{i} Q_{i}\left(\omega_{i}^{2}+\frac{d^{2}}{d t^{2}}\right) Q_{i}$

where it can be deduced from [1], see [37], that

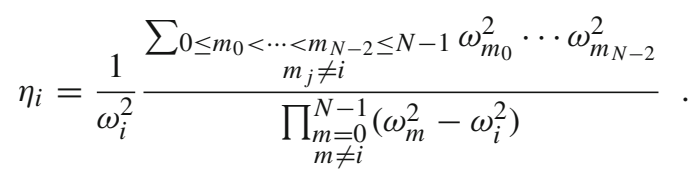

The signs of $\eta_{i}$ are alternating, which is a typical signature of HD dynamics, eventually leading to a total energy whose sign is undefined. The equation of motion for the $Q_{i}$ 's reads

$\ddot{Q}_{i}+\omega_{i}^{2} Q_{i}=0, \quad i=0, \ldots, N-1$.

Generalization of the $\mathrm{P}-\mathrm{U}$ oscillator in more than one spatial dimension is straightforward and will be left for future works. New integrals of motion such as HD angular momenta are naturally expected: We refer the interested reader to [3840] for explicit definitions.

\section{Hamiltonian formalism}

The Ostrograski construction that we have reviewed in Sect. 2.1 canonically leads to Hamiltonians that are not separable in the variables $p_{i}(2)$ and

$q^{i}:=x^{(i)}, \quad \forall i \in\{0, \ldots, N-1\}$

In this section we show in some particular cases that a suitable change of canonical variables allows to recast the $\mathrm{P}-\mathrm{U}$ Hamiltonian under a separable form.

\subsection{Ostrogradski’s approach}

As in the standard $N=1$ case, Hamilton's variational principle naturally leads to a symplectic structure and an Hamiltonian function $H$. Provided that the regularity condition (3) holds one can invert the last relation (2) defining the momentum $p_{N-1}$ in order to express $x^{(N)}$ in terms of $p_{N-1}$ and the $x^{(i)}$ 's, $i \in\{0, \ldots, N-1\}$ :

$$
\frac{\partial^{2} L}{\partial x^{(N)} \partial x^{(N)}} \neq 0 \Rightarrow x^{(N)}=\mathscr{V}\left(x^{(0)}, \ldots, x^{(N-1)}, p_{N-1}\right) \text {. }
$$


Details about singular HD Lagrangians can be found in [41, 42]. The Hamiltonian function $H$ is defined as

$$
\begin{aligned}
& H\left(\left\{q^{i}, p_{i}\right\}_{i=0, \ldots, N-1}\right):=\sum_{j=0}^{N-2} p_{i} q^{i+1} \\
& \quad+p_{N-1} \mathscr{V}-L\left(q^{0}, q^{1}, \ldots, q^{N-1}, \mathscr{V}\right) .
\end{aligned}
$$

The symplectic structure, as already apparent from the structure of the boundary terms in the variation $\delta S$ in the equation above (2), is given by the two-form $\boldsymbol{\Omega}=\sum_{i=0}^{N-1} d p_{i} \wedge d q^{i}$. In particular, the Poisson-Ostrogradski bracket between any two functions in the phase space $T^{*} \mathbb{Q}$ locally coordinatized by the $2 N$ variables $\left(q^{i}, p_{i}\right)_{i=0, \ldots, N-1}$ is given by

$\{f, g\}=\sum_{i=0}^{N-1}\left(\frac{\partial f}{\partial q^{i}} \frac{\partial g}{\partial p_{i}}-\frac{\partial f}{\partial p_{i}} \frac{\partial g}{\partial q^{i}}\right)$.

The symplectic structure $\boldsymbol{\Omega}$ amounts to writing $\left\{q^{i}, p_{j}\right\}=$ $\delta_{j}^{i},\left\{q^{i}, q^{j}\right\}=0=\left\{p_{i}, p_{j}\right\}, \forall i, j \in\{0, \ldots, N-1\}$, and the field equations are given as usual by $\dot{q}^{i}=\left\{q^{i}, H\right\} \equiv$ $\frac{\partial H}{\partial p_{i}}, \dot{p}_{i}=\left\{p_{i}, H\right\} \equiv-\frac{\partial H}{\partial q^{i}}$. The original equation of motion is reproduced from $\dot{p}_{0}=\left\{p_{0}, H\right\}$, while all the other equations above reproduce the relations (2) between the momenta- and the position-like variables.

Starting from Lagrangian (6) and applying Ostrogradski's procedure one gets

$p_{i}=\sum_{j=0}^{N-i-1}(-)^{j} a_{i+j+1} x^{(i+2 j+1)}, \quad i=0, \ldots, N-1$

and the Hamiltonian reads

$H=\sum_{j=0}^{N-2} p_{j} q^{j+1}+\frac{p_{N-1}^{2}}{2 a_{N}}-\frac{1}{2} \sum_{j=0}^{N-1} a_{j}\left(q^{j}\right)^{2}$.

The equations of motion are explicitly given by

$$
\begin{aligned}
\dot{q}^{i} & =q^{i+1}, \quad i=0, \ldots, N-2 \\
\dot{q}^{N-1} & =\frac{p_{N-1}}{a_{N}} \\
\dot{p}_{0} & =a_{0} q_{0} \\
\dot{p}_{j} & =-p_{j-1}+a_{j} q_{j}, \quad j=1, \ldots, N-1 .
\end{aligned}
$$

\subsection{Link with Pais-Uhlenbeck variables}

The equivalence of Ostrogradski and $\mathrm{P}-\mathrm{U}$ formalisms at Hamiltonian level is not obvious in the sense that the canonical transformation between the Ostrogradski and the P-U variables has not been explicitly given in the general case, as far as we could see. It was shown for $N=2$ in [31]. Here we show it for the $N=3$ case and leave the explicit form of the canonical transformation relating the Ostrogradski to the $\mathrm{P}-\mathrm{U}$ variables for future works. Below we simply give the general expression for the generating function of the canonical transformation for arbitrary $N$, without solving the partial differential equations that explicitly relate the two sets of phase space variables.

\subsubsection{The case $N=3$}

A convenient parametrisation in this case is

$L=-\frac{1}{2} x^{2}+\frac{A_{1}}{2 \Omega^{6}} \dot{x}^{2}-\frac{A_{2}}{2 \Omega^{6}} \ddot{x}^{2}+\frac{1}{2 \Omega^{6}} \dddot{x}^{2}$,

with

$A_{1}:=\sum_{i<j}\left(\omega_{i} \omega_{j}\right)^{2}, \quad A_{2}:=\sum_{i=0}^{2} \omega_{i}^{2}$,

$\Omega^{6}:=\left(\omega_{0} \omega_{1} \omega_{2}\right)^{2}$ and $\omega_{2}>\omega_{1}>\omega_{0}$.

Note that Lagrangian (22) can be recast under the form (12) provided that

$\sum_{i=0}^{2} \omega_{i}^{2} \eta_{i}=1, \quad \sum_{i=0}^{2} \omega_{i}^{4} \eta_{i}=0=\sum_{i=0}^{2} \omega_{i}^{6} \eta_{i}$.

The above constraints are fulfilled with (13). The momenta associated with (22) read

$p_{0}=\frac{1}{\Omega^{6}}\left(A_{1} \dot{x}+A_{2} \dddot{x}+x^{(5)}\right)$,

$p_{1}=-\frac{1}{\Omega^{6}}\left(A_{2} \ddot{x}+x^{(4)}\right), \quad p_{2}=\frac{\dddot{x}}{\Omega^{6}}$

and the Hamiltonian is given by

$$
\begin{aligned}
& H=p_{0} q^{1}+p_{1} q^{2}+\frac{\Omega^{6}}{2} p_{2}^{2}+\frac{1}{2}\left(q^{0}\right)^{2} \\
& -\frac{A_{1}}{2 \Omega^{6}}\left(q^{1}\right)^{2}+\frac{A_{2}}{2 \Omega^{6}}\left(q^{2}\right)^{2} .
\end{aligned}
$$

One can readily derive a generating function for the canonical transformation relating the Ostrogradski variables $\left(q^{0}, q^{1}\right.$, $\left.q^{2}, p_{0}, p_{1}, p_{2}\right)$ to the $\mathrm{P}-\mathrm{U}$ phase space variables $\left(Q_{0}, Q_{1}\right.$, $\left.Q_{2}, P_{0}, P_{1}, P_{2}\right)$. It is given by

$$
\begin{aligned}
F\left(q^{0}, p_{1}, q^{2}, P_{0}, P_{1}, P_{2}\right) & =\sum_{i=0}^{2} Q_{i}\left(q^{0}, p_{1}, q^{2}\right) P_{i} \\
& \equiv \sum_{i=0}^{2} \tilde{Q}_{i}\left(q^{0}, p_{1}, q^{2}\right) \tilde{P}_{i}
\end{aligned}
$$

It leads to the following system of partial differential equations:

$$
\begin{aligned}
& \frac{\partial F}{\partial P_{0}}=Q^{0}\left(q^{0}, p_{1}, q^{2}\right), \quad \frac{\partial F}{\partial P_{1}}=Q^{1}\left(q^{0}, p_{1}, q^{2}\right), \\
& \frac{\partial F}{\partial P_{1}}=Q^{1}\left(q^{0}, p_{1}, q^{2}\right),
\end{aligned}
$$


$\frac{\partial F}{\partial q^{0}}=p_{0}\left(q^{0}, p_{1}, q^{2}\right), \quad \frac{\partial F}{\partial p_{1}}=-q^{1}\left(q^{0}, p_{1}, q^{2}\right)$,

$\frac{\partial F}{\partial q^{2}}=p_{2}\left(q^{0}, p_{1}, q^{2}\right)$.

The first three equations are trivial, whereas the last three can be obtained from the relations $\sum_{i=0}^{2} \eta_{i} \omega_{i}^{2 m}=\delta_{1}^{m}, m=$ $1,2,3$, as well as $\sum_{i=0}^{2} \eta_{i} \omega_{i}^{8}=\omega_{0}^{2} \omega_{1}^{2} \omega_{2}^{2}$ and $\sum_{i=0}^{2} \eta_{i}=$ $\frac{A_{1}}{\Omega^{6}}$. In particular, it yields

$p_{0}=P_{0}+P_{1}+P_{2}, \quad p_{2}=-\frac{1}{\Omega^{6}} \sum_{i=0}^{2} \omega_{i}^{4} P_{i}$,

$q^{1}=\sum_{i=0}^{2} \omega_{i}^{2} P_{i}$

Recalling that the $\eta_{i}$ are of alternating sign, the Ostrogradski Hamiltonian eventually reads

$H=\frac{1}{2} \sum_{i=0}^{2}(-)^{i}\left(\frac{P_{i}^{2}}{\left|\eta_{i}\right|}+\left|\eta_{i}\right| \omega_{i}^{2} Q_{i}^{2}\right)$.

Let us observe that, from the expressions (25) for the Ostrogradski momenta and the definition (11) of the P-U oscillator variables $Q_{i}, i=0,1,2$, one has the relation $P_{i}=\eta_{i} \dot{Q}_{i}$, $i=0,1,2$. Finally, a last canonical transformation given by the mere rescaling $\tilde{Q}_{i}:=\sqrt{\left|\eta_{i}\right|} Q_{i}, \tilde{P}_{i}:=\frac{P_{i}}{\sqrt{\left|\eta_{i}\right|}}$, gives the following $\mathrm{P}-\mathrm{U}$ Hamiltonian:

$H=\frac{1}{2} \sum_{i=0}^{2}(-)^{i}\left(\tilde{P}_{i}^{2}+\omega_{i}^{2} \tilde{Q}_{i}^{2}\right)$.

\subsubsection{Arbitrary $N$}

The $N=2$ and $N=3$ cases show that Ostrogradski's procedure leads to $\mathrm{P}-\mathrm{U}$ Hamiltonian and variables by successive canonical transformations. Moreover Lagrangian (6), once expressed as (12), leads to the separable Hamiltonian

$H=\frac{1}{2} \sum_{i=0}^{N-1}(-)^{i}\left(\frac{P_{i}^{2}}{\left|\eta_{i}\right|}+\left|\eta_{i}\right| \omega_{i}^{2} Q_{i}^{2}\right)=: \sum_{i=0}^{N-1}(-)^{i} E_{i}$

for any $N$, where $E_{i}, i=0,1,2$ are positive definite quantities where

$Q_{i}:=\prod_{j \neq i}\left(1+\frac{1}{\omega_{j}^{2}} \frac{d^{2}}{d t^{2}}\right) x$,

$P_{i}:=\eta_{i} \dot{Q}_{i} \equiv(-)^{i}\left|\eta_{i}\right| \dot{Q}_{i}$,

and where the P-U coefficients $\eta_{i}$ can be found in [37] and in (13). Note that the frequencies $\omega_{k}$ are the roots of the polynomial equation $p\left(\omega_{k}\right)=0$, see $(8)$.

\section{Adiabatic invariants and Nekhoroshev estimates}

\subsection{Action variables}

The P-U Hamiltonian (33) is separable and admits elliptic trajectories in the planes $\left(Q_{i}, P_{i}\right), i \in\{0,1, \ldots, N-1\}$, these fixed- $E_{i}$ cycles being denoted as $\Gamma_{i}$. Hence a set of $N$ action variables can be defined:

$$
\begin{gathered}
I_{j}=\frac{(-)^{j}}{2 \pi} \oint_{\Gamma_{j}} P_{j} d Q_{j}=\frac{\left|\eta_{j}\right|}{2 \pi} \oint_{\Gamma_{j}} \dot{Q}_{j}^{2} d t, \\
j=0, \ldots, N-1 .
\end{gathered}
$$

The $(-)^{j}$ factor is such introduced in such a way that the action variables $\left\{I_{i}\right\}_{i=0, \ldots, N-1}$ are all positive. It can be checked that the Hamiltonian (33) reads

$H=\sum_{j=0}^{N-1}(-)^{j} I_{j} \omega_{j}, \quad$ with $\quad I_{j}=\frac{E_{j}}{\omega_{j}}$,

and that the relations

$I_{i}=\frac{\partial E_{i}}{\partial \omega_{i}}, \quad i=0, \ldots, N-1$

holds as well. The action variable $I_{0}$ reduces to the average kinetic energy for $N=1$, as expected.

The action variables can be expressed in terms of the classical trajectory $x(t)$ through the definition (34). When $N=2$ for example,

$I_{0} \sim \oint_{\Gamma_{0}}\left[\dot{x}^{2}+\frac{2}{\omega_{1}^{2}} \dot{x} \dddot{x}+\frac{1}{\omega_{1}^{4}} \dddot{x}^{2}\right] d t$,
$I_{1} \sim \oint_{\Gamma_{1}}\left[\dot{x}^{2}+\frac{2}{\omega_{0}^{2}} \dot{x} \dddot{x}+\frac{1}{\omega_{0}^{4}} \dddot{x}^{2}\right] d t$.

The cross-product $\dot{x} \dddot{x}$ cannot be expressed in terms of $\ddot{x}^{2}$ with the use of a total derivative since $x(t)$ is not a priori periodic with frequency $\omega_{0}$ or $\omega_{1}$ unless $\omega_{1} / \omega_{0}=$ $n / m \in \mathbb{Q}$. In the latter case, after a time $T=m 2 \pi / \omega_{0}=$ $n 2 \pi / \omega_{1}$, the action variables can be recast under the form $\oint_{\Gamma_{0}}\left[\dot{x}^{2}+c \ddot{x}^{2}+d \dddot{x}^{2}\right] d t$ with $c, d$ real coefficients. At the same time, the commensurability condition on $\omega_{1} / \omega_{0}$ implies instability of the $N=2$ dynamics under small perturbations so it is not relevant for the present study [31].

The $I_{j}$ are constant of motion provided that $H$ does not explicitly depend on time. It is nevertheless possible that some external parameter is time-dependent: we set $\omega_{i}=$ $\omega_{i}(t)$ in (33). Under the assumption that the $\omega_{i}(t)$ vary slowly enough with respect to the typical duration of a cycle - and despite the fact that no rigorous definition of "enough" can be given [43] -, the quantities $I_{j}$ given by (36) are adiabatic invariants. It can be deduced from [44] that their small rate of change is given by: 
$\dot{I}_{k}=-\frac{\dot{\omega}_{k}}{\omega_{k}} I_{k} \cos 2 \phi_{k}$

$\dot{\phi}_{k}=(-)^{k} \omega_{k}+\frac{\dot{\omega}_{k}}{2 \omega_{k}} \sin 2 \phi_{k}$,

where $\phi_{k}$ are the angle variables conjugated to $I_{k}:\left\{\phi_{j}, I_{k}\right\}=$ $\delta_{j k}$. The interested reader may find general computations related to time-varying harmonic oscillators in [45-47].

As an illustration of the above relations, suppose that the time-dependent parameter is a small perturbation of the frequencies:

$\omega_{i}(t)=\varpi_{i}\left(1+\epsilon g_{i}(t)\right)$, with $|\epsilon| \ll 1$,

the only dependence on time being contained in the real functions $g_{i}$. At the lowest order in $\epsilon$, Eqs. (40) become

$\dot{I}_{k}=-\epsilon \dot{g}_{k} I_{k} \cos 2 \varphi_{k}$,

$\dot{\phi}_{k}=(-)^{k} \varpi_{k}\left(1+\epsilon g_{k}\right)+\frac{\epsilon}{2} \dot{g}_{k} \sin 2 \varphi_{k}$,

$\varphi_{k}:=(-)^{k} \varpi_{k} t+\alpha_{k}$

For $g_{i}(t)$ arbitrary and $\bar{I}_{k}$ the values of the action variables for the unperturbed system, the simple shape

$I_{k}=\bar{I}_{k}\left(1-\epsilon g_{k}\right)$

solves Eq. (42) at the first order in $\epsilon$ when $\varphi_{k}=n \pi, n \in \mathbb{Z}$. It may therefore be used to estimate the trend of the modifications induced by $g_{i}$ on the action variables. In the special case $g_{i}(t)=\bar{g} \sin \left(\Omega_{i} t+\beta_{i}\right)$, the solution of (42) up to first order in $\epsilon$ is given by

$$
\begin{aligned}
I_{k}= & \bar{I}_{k}\left[1-\frac{\epsilon}{2} \bar{g}\left(\frac{\Omega_{k}}{2 \omega_{k}^{+}} \sin 2\left(\omega_{k}^{+} t+\alpha_{k}^{+}\right)\right.\right. \\
& \left.\left.+\frac{\Omega_{k}}{2 \omega_{k}^{-}} \sin 2\left(\omega_{k}^{-} t+\alpha_{k}^{-}\right)\right)\right], \\
\phi_{k}= & \alpha_{k}+(-)^{k} \varpi_{k} t+(-)^{k+1} \epsilon \frac{\varpi_{k}}{\Omega_{k}} \bar{g} \cos \left(\Omega_{k} t+\beta_{k}\right) \\
& +(-)^{k+1} \frac{\epsilon}{4} \bar{g}\left(\frac{\Omega_{k}}{2 \omega_{k}^{+}} \cos 2\left(\omega_{k}^{+} t+\alpha_{k}^{+}\right)\right. \\
& \left.+\frac{\Omega_{k}}{2 \omega_{k}^{-}} \cos 2\left(\omega_{k}^{-} t+\alpha_{k}^{-}\right)\right), \\
\omega_{k}^{ \pm}:= & \varpi_{k} \pm \frac{(-)^{k}}{2} \Omega_{k}, \quad \alpha_{k}^{ \pm}:=(-)^{k}\left(\alpha_{k} \pm \frac{\beta_{k}}{2}\right) .
\end{aligned}
$$

In the case where the perturbations $g_{k}(t)$ are slow, as is prescribed by the theory of adiabatic invariants, one has that the frequencies $\Omega_{k}$ are small compared to the characteristic frequencies $\varpi_{k}$ of the system and one finds that the variation of the action variables starts at second order, while the variation of the angle variables starts earlier.

\subsection{Nekhoroshev estimates}

The P-U Hamiltonian (37) with the time-dependent perturbation (41) can be formally written under the form

$H=h(\boldsymbol{I})+\epsilon f(\boldsymbol{I}, \boldsymbol{\phi})$,

where

$h(\boldsymbol{I})=\varpi \cdot \boldsymbol{I}, \quad f(\boldsymbol{I}, \boldsymbol{\phi})=\boldsymbol{I} \cdot \boldsymbol{g}(\boldsymbol{\phi})$,

and where the vectors $(\boldsymbol{I})_{k}=I_{k},(\boldsymbol{\phi})_{k}=\phi_{k},(\boldsymbol{g})_{k}=g_{k}$ and $(\varpi)_{k}=(-)^{k} \varpi_{k}$ have been introduced.

Nekhoroshev's theorem [32,33] states that if the nearly integrable Hamiltonian (45) is analytic and the unperturbed part $h(\boldsymbol{I})$ is steep (or convex, or quasiconvex) on some domain, then there is a threshold $\epsilon_{0}>0$ and positive constants $R, T, a$ and $b$ such that whenever $|\epsilon|<\epsilon_{0}$, for all initial actions variables $\boldsymbol{I}(0)$ in the domain (and far enough from the boundary), one has

$\max \left\{\left|I_{k}(t)-I_{k}(0)\right|\right\}<R \epsilon^{b}$ for times $|t|<T \exp \varepsilon^{-a}$.

This result has been particularized to several explicit examples, among which the harmonic oscillator in Refs. [29,30]. The fact that some components of $\varpi$ are negative is allowed by the formalism of the latter references. From the study [29] in particular, it can be deduced that unstable behaviours in the $\mathrm{P}-\mathrm{U}$ oscillator appears at exponentially large time in $\epsilon$, i.e. the dynamics is classically stable. Since $\epsilon_{0} \sim N^{-2 N}$ [29], the more the dynamics contains HD, the more the perturbation must be small to preserve stability. Moreover, there must exist two real positive constants $\sigma, \tau$ such that $|\varpi \cdot \boldsymbol{n}| \geq \sigma\left[\sum_{j=0}^{N-1}\left|n_{j}\right|\right]^{-\tau}$ for all $\boldsymbol{n} \in \mathbb{Z}_{0}^{N}$ otherwise $\epsilon_{0}$ becomes arbitrarily large and the system is unstable. In other words the frequencies must define a nonresonant harmonic oscillator. Such an instability can only occur for $N>1$ onedimensional dynamics; it is trivially avoided when $N=1$ because no energy transfer between the different components $E_{i}$ are de facto absent in this case.

\section{Concluding comments}

Higher-derivative action principles generally lead to unstable classical dynamics. However, all the classical trajectories allowed by the Pais-Uhlenbeck oscillator (10) with distinct and nonresonant frequencies are bounded: it is an explicit realisation of a stable classical theory with higherderivatives. Therefore the problem can be formulated in action-angle variables formalism, allowing a computation of adiabatic invariants and a proof of the classical stability based on Nekhoroshev estimates. Although the Pais- 
Uhlenbeck oscillator has been widely studied as a prototypal higher-derivative physical theory, it is the first time, to our knowledge, that such results are obtained. Emphasis has been put on harmonic potentials in the present study. Other types of potentials or higher-derivative terms may also lead to stable classical dynamics. For example it is shown in Ref. [31] that a $N=2$ Pais-Uhlenbeck oscillator with cubic and quartic potential terms is stable too, except for very special values of the parameters. The stable models of [31] should give, after generalisation to field theory Lagrangians, further examples (compared to the one reviewed in [10]) of wellbehaved dynamical systems with infinite number of degrees of freedom.

One can recover a Lagrangian function from a Hamiltonian function provided that the regularity condition det $\frac{\partial^{2} H}{\partial p_{N-1} \partial p_{N-1}} \neq 0$ is satisfied - which is the case for a free system that is regular when the added interactions in the perturbed Hamiltonian are algebraic functions in the $\mathrm{P}-\mathrm{U}$ variables $Q$ 's, as we assumed implicitly here. This is clear for $N$ odd since the latter variables do not imply Ostrogradski's last momentum $p_{N-1}$. In the even $N$ cases where the $Q$ variables involve $p_{N-1}$, the added perturbations cannot ruin the regularity property of the unperturbed $\mathrm{P}-\mathrm{U}$ Hamiltonian as long as the perturbation is of polynomiality degree in $Q$ higher than two, as we assume here. However, it is notoriously difficult (see e.g. Section 8 of [10]) to find the analytical expression of the perturbed Lagrangian corresponding to a given Hamiltonian perturbation. It it usually nonlocal, even for simple (e.g. quartic) Hamiltonian perturbation [10], which suggests considering as a starting point the $N \rightarrow \infty$ limit studied by Pais and Uhlenbeck, as it would encompass all the possible Hamiltonian perturbations.

It is worth making comments about quantization. In a first approximation a Bohr-Sommerfeld quantization rule can be applied since action variables exist. The fact that the energy spectrum is unbounded both from below and above in higherderivative theories does not a priori forbids well-behaved quantum dynamics. In fact, a quantization technique was proposed in [6] that keeps the higher-derivative dynamics stable at the quantum level. In fact, we propose that the positivedefinite quantities suggested in [6] and that are responsible for a stable dynamics at the quantum level are nothing but the action variables. Indeed, even for a perturbatively perturbed classical motion, as long as the trajectories are bounded, the action variables are positive-definite quantities and conserved to the approximation given. More recently, it was conjectured in [10] that indeed, when all the classical trajectories of a given higher-derivative model are bounded, its quantum dynamics only contains so-called benign ghosts, i.e. negative-energy quantum states with a normalisable wave function and preserved unitarity of the evolution. The present work aimed at clarifying the conditions for higher-derivative models to exhibit bounded classical dynamics; hence it is a step toward the identification of quantum models with unitary quantum dynamics, to which the Pais-Uhlenbeck oscillator belongs. Further issues about quantization of higher-derivative Lagrangians are discussed for example in [48-50].

Finally it has to be noticed that the necessary conditions for adiabatic invariants and Nekhoroshev estimates to be computed are the separability of the higher-derivative Hamiltonian and the existence of bounded classical trajectories. Both conditions are met in the Pais-Uhlenbeck oscillator case after appropriate choice of canonical variables, but we believe that other classes of higher-derivative systems may be studied by resorting to the methods we have presented.

Data Availability Statement This manuscript has no associated data or the data will not be deposited. [Authors' comment: The paper contains formal developments in theoretical Physics. There are no data associated to them.]

Open Access This article is distributed under the terms of the Creative Commons Attribution 4.0 International License (http://creativecomm ons.org/licenses/by/4.0/), which permits unrestricted use, distribution, and reproduction in any medium, provided you give appropriate credit to the original author(s) and the source, provide a link to the Creative Commons license, and indicate if changes were made. Funded by $\mathrm{SCOAP}^{3}$.

\section{References}

1. A. Pais, G.E. Uhlenbeck, On Field theories with nonlocalized action. Phys. Rev. 79, 145 (1950)

2. V.V. Nesterenko, On the instability of classical dynamics in theories with higher derivatives. Phys. Rev. D 75, 087703 (2007). hep-th/0612265

3. A.V. Smilga, Comments on the dynamics of the Pais-Uhlenbeck oscillator. SIGMA 5, 017 (2009). arXiv:0808.0139

4. E. Joung, K. Mkrtchyan, Higher-derivative massive actions from dimensional reduction. JHEP 1302, 134 (2013). arXiv:1212.5919

5. M. Pavi, Pais-Uhlenbeck oscillator with a benign friction force. Phys. Rev. D 87(10), 107502 (2013). arXiv:1304.1325

6. D.S. Kaparulin, S.L. Lyakhovich, A.A. Sharapov, Classical and quantum stability of higher-derivative dynamics. Eur. Phys. J. C 74(10), 3072 (2014). arXiv:1407.8481

7. R.P. Woodard, Ostrogradsky's theorem on Hamiltonian instability. Scholarpedia 10(8), 32243 (2015). arXiv: 1506.02210

8. I. Masterov, The odd-order Pais-Uhlenbeck oscillator. Nucl. Phys. B 907, 495 (2016). arXiv: 1603.07727

9. M. Avendao-Camacho, J.A. Vallejo, Y. Vorobiev, A perturbation theory approach to the stability of the Pais-Uhlenbeck oscillator. J. Math. Phys 58(9), 093501 (2017). arXiv:1703.08929

10. A. Smilga, Classical and quantum dynamics of higher-derivative systems. Int. J. Mod. Phys. A 32(33), 1730025 (2017). arXiv: 1710.11538

11. S. Nicolis, Higher time derivatives in the microcanonical ensemble describe dynamics of flux-coupled classical and quantum oscillators (2018). arXiv: 1805.07934 [hep-th]

12. P.A. Horvathy, M.S. Plyushchay, Non-relativistic anyons, exotic Galilean symmetry and noncommutative plane. JHEP 0206, 
033 (2002). https://doi.org/10.1088/1126-6708/2002/06/033. hepth/0201228

13. M.S. Plyushchay, Majorana equation and exotics: higher derivative models, anyons and noncommutative geometry. Electron. J. Theor. Phys 3(10), 17 (2006). math-ph/0604022

14. K.S. Stelle, Renormalization of higher derivative quantum gravity. Phys. Rev. D 16, 953 (1977)

15. K.S. Stelle, Classical gravity with higher derivatives. Gen. Relativ. Gravit. 9, 353 (1978)

16. M. Nitta, R. Yokokura, Higher derivative three-form gauge theories and their supersymmetric extension. JHEP 1810, 146 (2018). arXiv: 1809.03957

17. G. Giribet, O. Miskovic, R. Olea, D. Rivera-Betancour, Energy in higher-derivative gravity via topological regularization. Phys. Rev. D 98, 044046 (2018). arXiv:1806.11075

18. E. Tomboulis, Renormalizability and asymptotic freedom in quantum gravity. Phys. Lett. B 97, 77-80 (1980)

19. H.J. Schmidt, Stability and Hamiltonian formulation of higher derivative theories. Phys. Rev. D 49, 6354 (1994). Erratum: [Phys. Rev. D 54 (1996) 7906] gr-qc/9404038

20. S.W. Hawking, T. Hertog, Living with ghosts. Phys. Rev. D 65, 103515 (2002). hep-th/0107088

21. S. Ferrara, A. Kehagias, D. Lst, Aspects of Weyl supergravity. JHEP 1808, 197 (2018). arXiv:1806.10016

22. A.R.R. Castellanos, F. Sobreira, I.L. Shapiro, A.A. Starobinsky, On higher derivative corrections to the $R+R^{2}$ inflationary model. JCAP 1812(12), 007 (2018). arXiv: 1810.07787

23. W.L. Nelson, Physical principles for economics of skilled movements. Biol. Cybern. 46, 135-147 (1983)

24. N. Hogan, An organizing principle for a class of voluntary movements. J. Neurosci. 4, 2745-2754 (1984)

25. S. Lebedev, W.H. Tsui, P. Van Gelder, Drawing movements as an outcome of the principle of least action. J. Math. Psychol. 45, 43-52 (2001)

26. S. Hagler, On the principled description of human movements (2015). arXiv:1509.06981

27. D. Huh, T.J. Sejnowski, Conservation law for self-paced movements. PNAS 113, 8831-8836 (2016)

28. M. Ostrogradsky, Mémoires sur les équations différentielles, relatives au problème des isopérimètres. Mem. Acad. St. Petersbourg 6(4), 385 (1850)

29. G. Benettin, G. Gallavotti, Stability of motions near resonances in quasi-integrable Hamiltonian systems. J. Stat. Phys. 44, 293-338 (1986)

30. E. Valdinoci, Estimates for non-resonant normal forms in Hamiltonian perturbation theory. J. Stat. Phys. 101, 4 (2000)

31. E. Pagani, G. Tecchiolli, S. Zerbini, On the problem of stability fir higher-order derivative Lagrangian systems. Lett. Math. Phys. 14, 311-319 (1987)
32. N.N. Nekhoroshev, Behavior of Hamiltonian systems close to integrable. Funct. Anal. Appl. 5, 338-339 (1971)

33. N.N. Nekhoroshev, An exponential estimate of the time of stability of nearly-integrable Hamiltonian systems. Russ. Math. Surv. 32, $1-65$ (1977)

34. A.V. Smilga, Ghost-free higher-derivative theory. Phys. Lett. B 632 , 433 (2006). hep-th/0503213

35. H. Motohashi, K. Noui, T. Suyama, M. Yamaguchi, D. Langlois, Healthy degenerate theories with higher derivatives. JCAP 1607, 033 (2016). arXiv: 1603.09355

36. N.G. Stephen, On the Ostrogradski instability for higher-order derivative theories and a pseudo-mechanical energy. J. Sound Vib. 310, 729 (2008)

37. S.V. Ketov, G. Michiaki, T. Yumibayashi, Quantizing with a higher time derivative, advances in quantum field theory, InTech Publishers 2012, pp. 49-73. arXiv:1110.1155 [hep-th]

38. M. Borneas, Principle of action with higher derivatives. Phys. Rev. 186, 1299 (1969)

39. F. Riahi, On Lagrangians with higher order derivatives. Am. J. Phys. 40, 386 (1972)

40. G.C. Constantelos, Integrals of motion for Lagrangians including higher-order derivatives. Nuovo Cim. B 21, 279 (1974)

41. V.V. Nesterenko, The singular Lagrangians with higher derivatives. J. Phys. A 22, 1673 (1989)

42. P. Dunin-Barkowski, A. Sleptsov, Geometric Hamiltonian formalism for reparametrization invariant theories with higher derivatives. Theor. Math. Phys. 158, 61 (2009). arXiv:0801.4293 [hep-th]

43. J.H. Hannay, Angle variable holonomy in adiabatic excursion of an integrable Hamiltonian. J. Phys. A Math. Gen. 18, 221-230 (1985)

44. L. Landau, E. Lifchitz, Physique théorique Tome 1 : Mécanique (4th Ed., MIR Moscou, 1988)

45. C.G. Wells, S.T.C. Siklos, The adiabatic invariance of the action variable in classical dynamics. Eur. J. Phys. 28, 105-112 (2007). arXiv:physics/0610084v1

46. L.L. Snchez-Soto, J. Zoido, Variations on the adiabatic invariance: the Lorentz pendulum. Am. J. Phys. 81, 57 (2012)

47. R.M. Kulsrud, Adiabatic invariant of the Harmonic oscillator. Phys. Rev. 106, 205-207 (1957)

48. D.M. Gitman, S.L. Lyakhovich, I.V. Tyutin, Hamilton formulation of a theory with high derivatives. Sov. Phys. J. 26, 730-734 (1983)

49. J.Z. Simon, Higher derivative Lagrangians, nonlocality, problems and solutions. Phys. Rev. D 41, 3720 (1990)

50. C. Grosse-Knetter, Effective Lagrangians with higher derivatives and equations of motion. Phys. Rev. D 49, 6709-6719 (1994) 